\title{
Research Paper \\ Projective Response of Patients With Somatic Symptom Disorder Compared To Controls Based On Location Score in Rorschach Test
}

\author{
Zeinab Abdolhosseini ${ }^{1}{ }^{10},{ }^{*}$ Isaac Rahimian Boogar ${ }^{1}\left(\mathbb{D}\right.$, Siyavash Telepasand $^{2}$ (1)
}

1. Department of Clinical Psychology, Faculty of Psychology and Educational Sciences, Semnan University, Semnan, Iran

2. Department of Educational Psychology, Faculty of Psychology and Educational Sciences, Semnan University, Semnan, Iran.

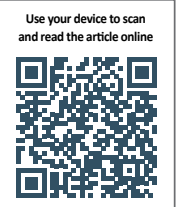

Citation: Abdolhosseini Z, Rahimian Boogar I, Telepasand S. [Projective Response of Patients with Somatic Symptom Disorder Compared To Controls Based On Location Score in Rorschach Test (Persian)]. Journal of Arak University of Medical Sciences (JAMS). 2020; 22(6):262-273. https://doi.org/10.32598/JAMS.22.6.5966.1

doi) $h$ https://doi.org/10.32598/JAMS.22.6.5966.1

Key words:

Somatic symptom disorder, Rorschach test, Perceived location

\section{ABSTRACT}

Article Info:

Received: 10 Aug 2019 Accepted: 12 Nov 2019 Available Online: 01 Feb 2020
Background and Aim Physical complaints that have no clear cause are a process that is commonly seen in patients with Somatic Symptom Disorder (SSD). The increasing number of patients with this disorder and its related chronic symptoms including changes in quality of life and loss of individual efficiency has created social and psychological economic costs for these patients and their families.

Methods \& Materials This descriptive cross-sectional study conducted on 70 patients with SSD having digestive symptoms and chronic pain referred to the health centers in Semnan, Iran in winter 2018. They were selected using a convenience sampling method. The data collection tools were 15-item Patient Health Questionnaire Physical Symptoms (PHQ-15) and Rorschach test (Exner's Comprehensive System). In order to analyze the collected data, multiple linear regression analysis, logistic regression analysis, and multivariate ANOVA were performed in SPSS V. 22 software.

Ethical Considerations The present study was approved by the Research Ethics Committee of Semnan University of Medical Sciences (Code: IR.SEMUMS.REC.1397.029).

Results Perceived location indices including $D$ and $D d$ scores in Rorschach test were able to significantly differentiate the SSD patients from controls $(P<0.05)$. In the logistic regression model, $70 \%$ of participants were classified properly.

Conclusion There is difference in projective responses between SSD patients and controls, and patients were less holistic. Anxiety and psychiatric symptoms were also higher in patients with SSD.

\section{Extended Abstract}

\section{Introduction}

I

$\mathrm{n}$ the last few decades, a type of mental disorders called Somatic Symptom Disorder (SSD) has emerged that is affected by emotional and psychological factors [2]. Patients with SSD usually have multiple physical symptoms that are unpleasant or lead to significant disruption in daily living and may not be medically justified [2]. Physical complaints that do not have specific causes are a process commonly seen in patients with SSD. People with this disorder have no view of the psychological symptoms of their illness and refuse to undergo psychiatric clinical therapies [7]. Due to the complexity of experiences in SSD, projective personality tests can be used to provide multidimensional psychological aspects of patients with this disorder [12]. The main purpose of this study was to investigate the differences

\section{* Corresponding Author:}

Isaac Rahimian Boogar, PhD.

Address: Department of Clinical Psychology, Faculty of Psychology and Educational Sciences, Semnan University, Semnan, Iran.

Tel: +98 (23) 33624250

E-mail: i_rahimian@semnan.ac.ir 
between the projective responses of patients with and without SSD using Rorschach test in order to suggest useful psychological approaches to help patients with SSD and be used to treat and diagnose these patients.

\section{Methods and Materials}

This is a analytical study with cross-sectional design. The statistical population included all men and women aged 20-60 years with gastrointestinal disease and physical pain referred to the health centers of in Semnan, Iran in 2018. Of these, 70 were selected as study samples by using a convenience sampling technique and based on inclusion and exclusion criteria and divided into two groups of patients $(n=35)$ and controls $(n=35)$. The data collection tools were 15-item Patient Health Questionnaire Physical Symptoms (PHQ-15) and Rorschach test (Exner's Comprehensive System). After receiving the initial diagnosis report from the physician, the patients completed the PHQ-15 and after a semi-structured interview, Rorschach test was performed by each patient.

The psychometric properties of PHQ-15 have already been evaluated by Abdolmohammadi et al. Concurrent validity of this questionnaire with somatization subscale of the Symptom Checklist 90-item version (SCL-90) was $74 \%$, and its internal consistency using Cronbach's alpha was 0.76 [20]. Rorschach test was designed by Hermann Rorschach, a Swiss psychiatrist [21]. It is a performancebased personality test that is interpreted based on individual responses to 10 symmetrical inkblots [22]. In order to analyze the collected data, multiple linear regression analysis, logistic regression analysis, and multivariate ANOVA were performed in SPSS v. 22 software.

\section{Results}

Affected and non-affected groups were compared in terms of educational level, gender, employment, education and other factors. Out of 70 samples, 67.1\% were female. Their mean age was $34 \pm 3.51$ years (ranged $21-57$ years). Out of 35 people in the affected group, the PHQ15 score of 18 was ranged 35-45 and for 12 samples, it was 30-35. The PHQ-15 score was less than 30 in control group. The logistic regression model included five location variables based on Rorschach test (W, D, Dd, Ds and Dds ). The results of the Hosmer-Lemeshow test as the most reliable goodness-of-fit test in the logistic regression, showed a chi-square value of $3.98(\mathrm{Df}=8, \mathrm{P}=0.85)$. According to the results shown in Table 2, perceived location codes including D and Dd scores were able to significantly differentiate the SSD patients from controls $(\mathrm{P}<0.05)$.

\section{Discussion}

Statistical results showed that there was a significant difference between participants' projective responses (affected and non-affected) to Rorschach test cards in terms of location variables, and perceived location scores played a predictive role in separating the SSD patients from nonaffected groups. Among the location variables, the D and Dd scores were more important in showing the response difference. In the interpretation of Exner's Comprehensive System, D scores are compared with W scores.

The $\mathrm{W}$ score reflects an overall view of the individual and having a more comprehensive view. In this regard, it can be justified that individuals with SSD may be less comprehensive and the high frequency of Dd scores in them may indicate symptoms such as anxiety and obsessive-compulsive behaviors that were reduced due to their response details. The results of Yamamoto et al.'s study also showed that the perceived location scores of two groups of schizophrenia and autism were significantly different and those with schizophrenia had higher D scores [17]. Contrary to our results, Pasha Sharifi and Kheradpey in a study with the purpose of comparing the responses of depressed and anxious individuals with healthy people using Rorschach test, showed no significant difference between the scores of anxiety and depressed individuals in terms of Rorschach test variables [19].

SSD can be effective in choosing the location of perception and response to Rorschach test cards. In fact, if people are stressed and anxious, it will affect how they respond and pay attention to details. The results of this study, while confirming the importance of Rorschach test in the diagnosis of disorders that are affected by psychological factors, can be effective in designing treatment plans for people with disorders such as SSD. Since the results indicated high levels of anxiety in these patients, psychotherapy should also be considered in these patients' treatment plans.

\section{Ethical Considerations}

\section{Compliance with ethical guidelines}

The present study was approved by the Research Ethics Committee of Semnan University of Medical Sciences (code: IR.SEMUMS.REC.1397.029). 


\section{Funding}

This article is taken from the bachelor's thesis of the first author in the field of clinical psychology, Faculty of Psychology and Educational Sciences, Semnan University.

Authors' contributions

All authors met the writing standards based on the recommendations of the International Committee of Medical Journal Editors (ICMJE), and they had equal attribution in preparing the paper.

Conflicts of interest

The authors declared no conflict of interest. 


\title{
ياسخحاى فرافكن مبتلايان به اختلال علائم جسمى در مقايسه با افراد غيرمبثلا بر اساس نمرات محل ادراى آزمون رورشاخ ميثان
}

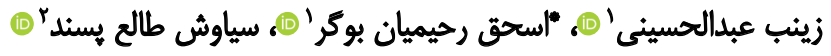

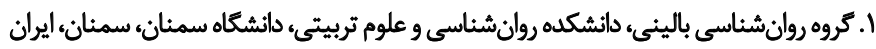

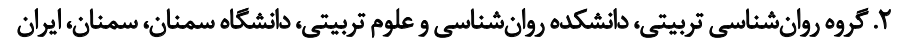

\begin{abstract}
جيكيد

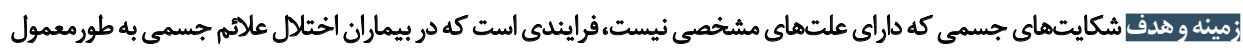

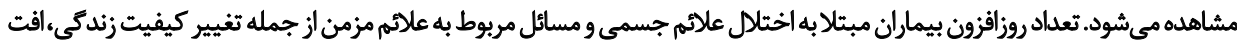

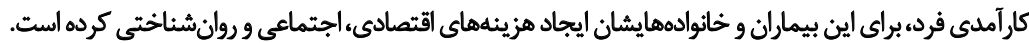

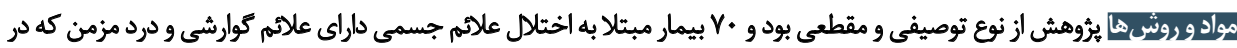

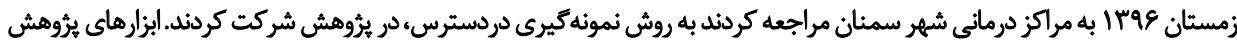

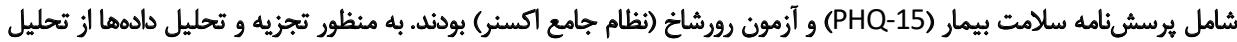

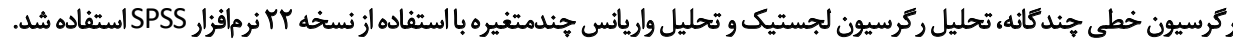

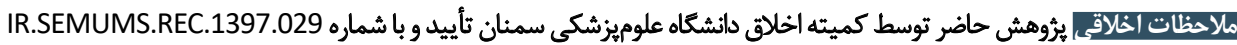
به ثبث رسيد.

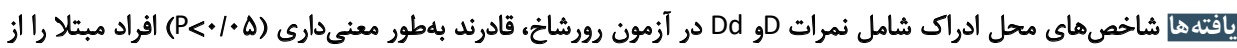

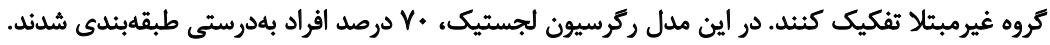

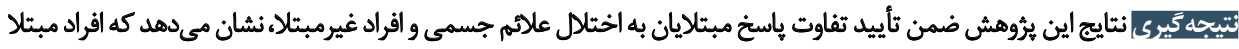

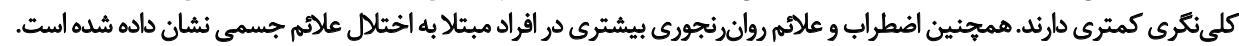

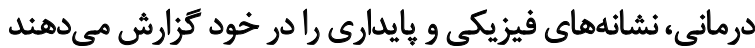

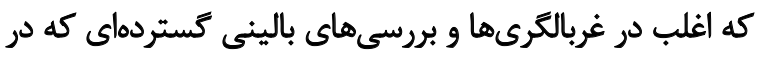

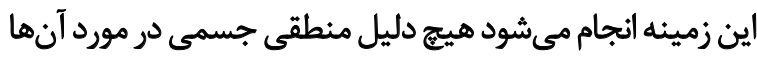

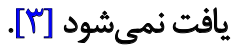

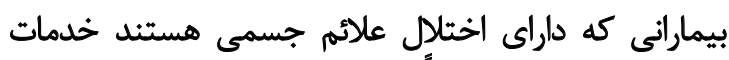

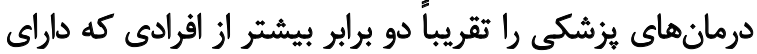

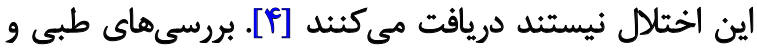

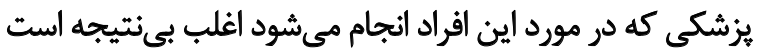

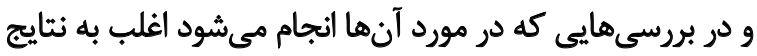

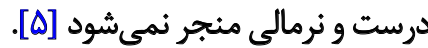

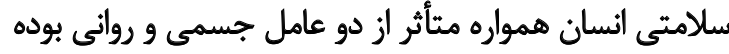

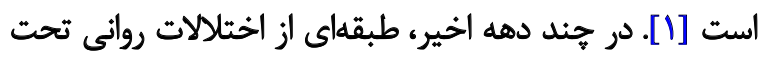

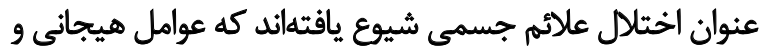

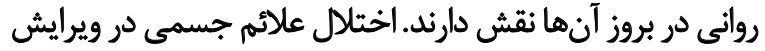

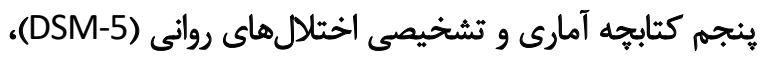

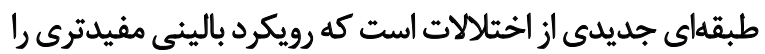

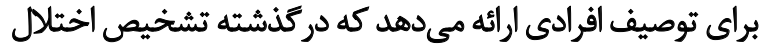
جسمانى سازى دريافت مي كردند.

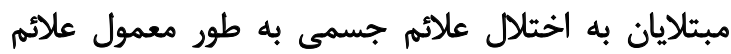

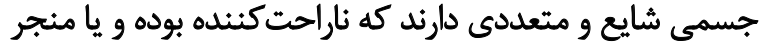

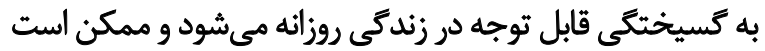

* نويسئده مسئول:

اسيحق رحيميان بوكر

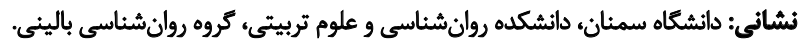
تلفن: +

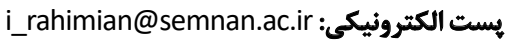




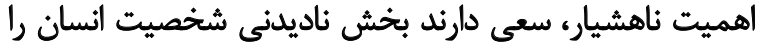

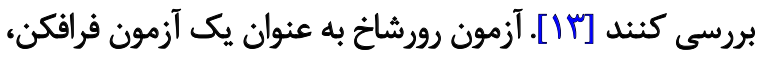

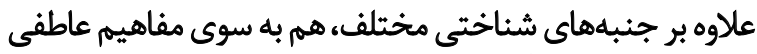

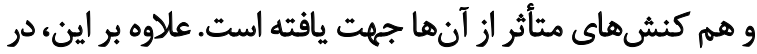

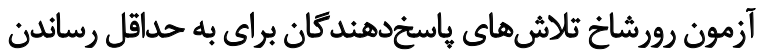

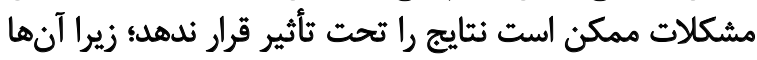

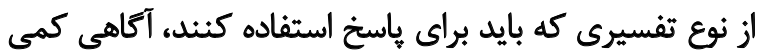

دارند [if]

فرض اصلى رورشاخ اين بود كه محركهاى محيطي به إنهائ

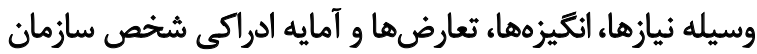

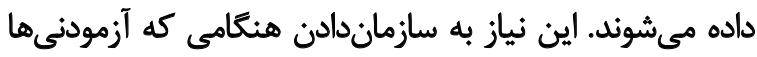

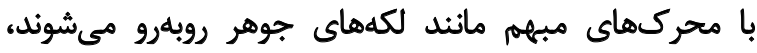

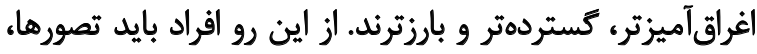

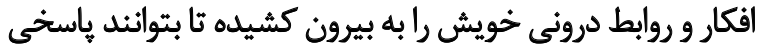

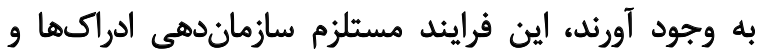

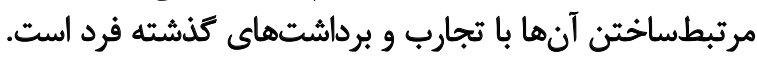

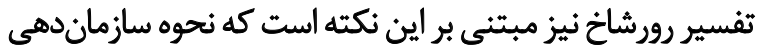

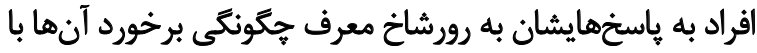

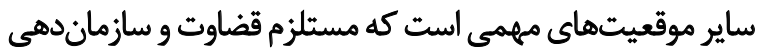

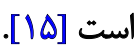

يكى از مزيتهاى رورشاخ كه اغلب به آن اشاره ميشود اين

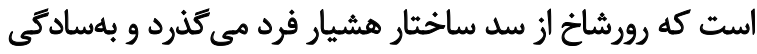

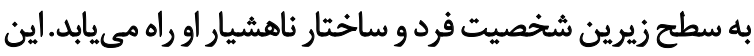

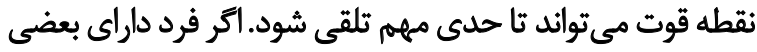

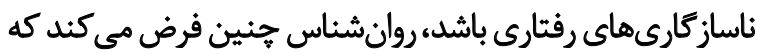

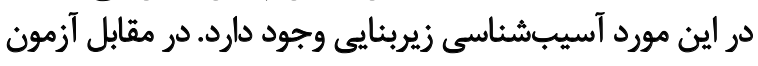

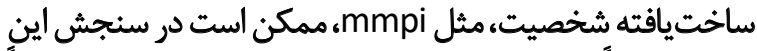

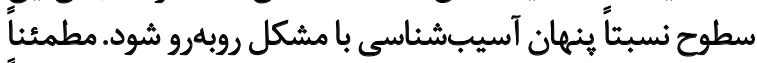

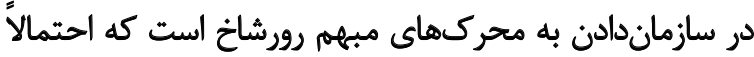

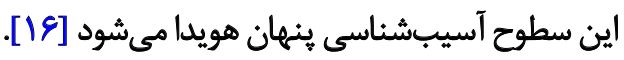

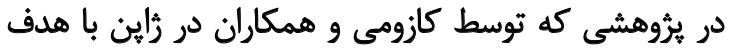

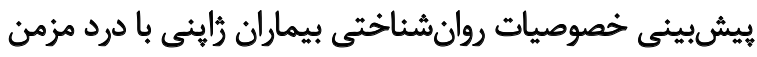

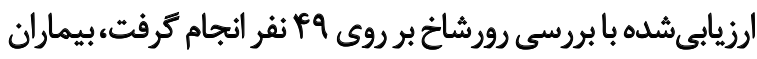

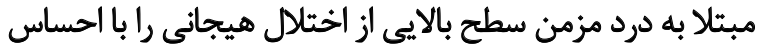

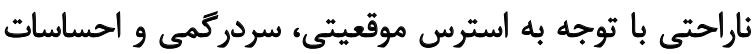

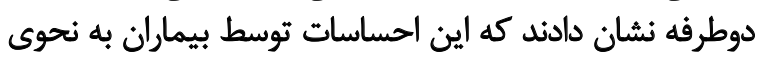

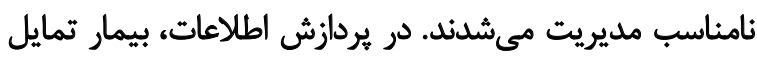

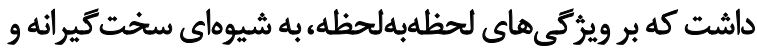

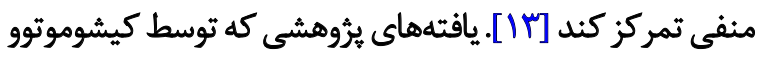

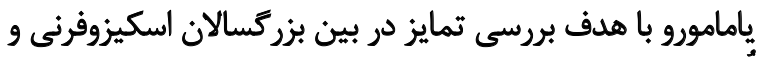

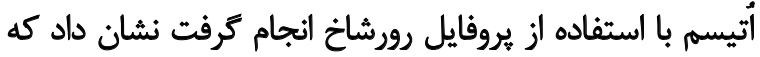

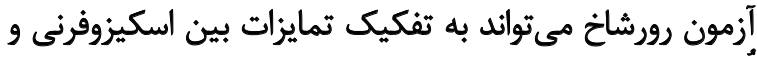

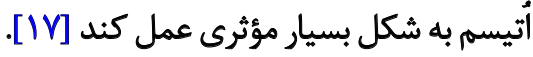

آسيبشناسىهاي روانى نشان ميدهد كه اين دسته از بيماران

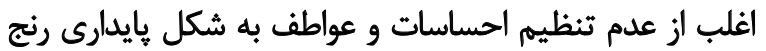

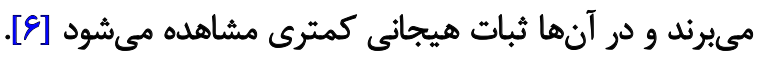

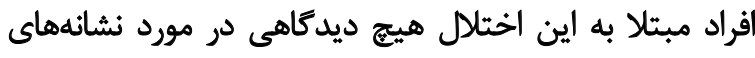

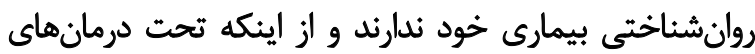

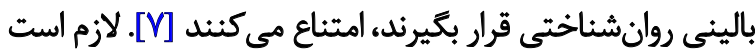

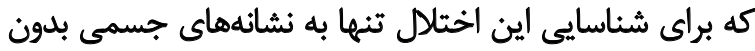

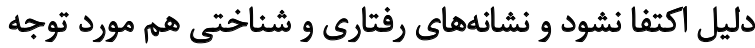

قرار تيرد [1]].

در DSM-5 يك دسته از فرايندهاى روانشناختى به عنوان

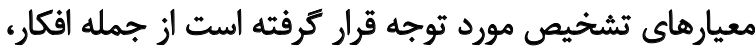

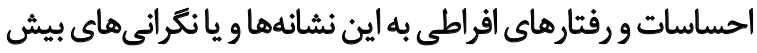

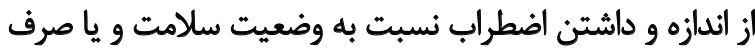

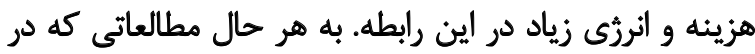

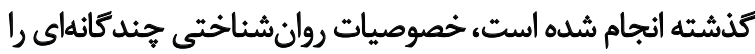

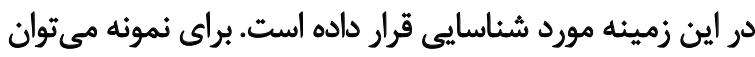

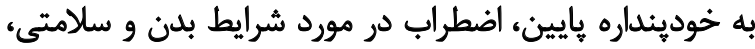

[اشاره كرد [9].]

بيمارانى كه از اختلال علائم جسمى رنج مىبرند آسيبهاي

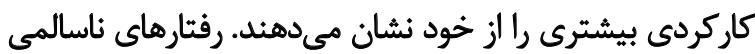

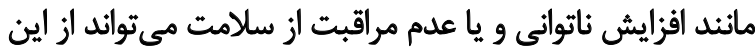

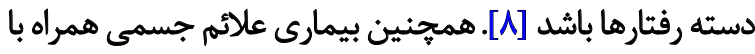

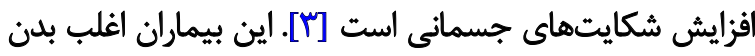

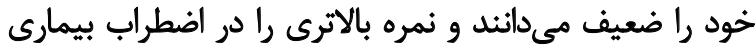

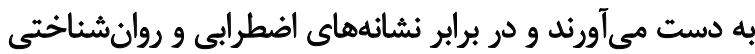

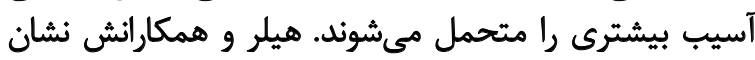

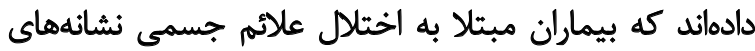

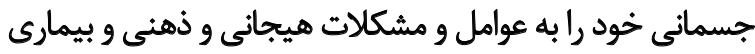

جسمى نسبت مى دهند [• (1]

خصوصيات رفتارى و روانشناختى كونائونى وجود دارد كه

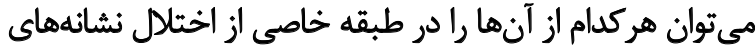

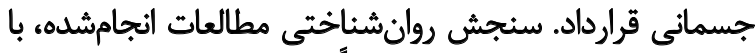

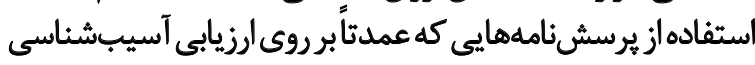

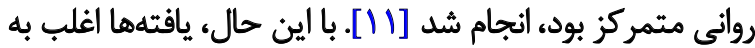

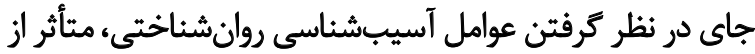

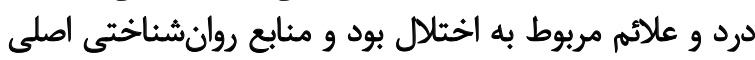

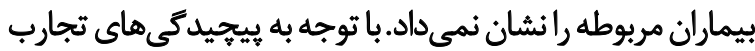

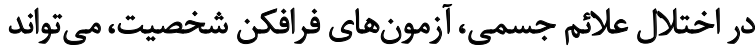

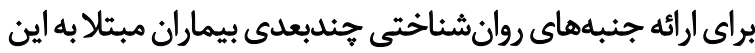

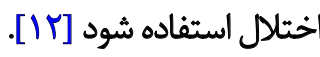

روانشناسان بالينى، آزمونهاى فرافكن شخصيت را براي الفي

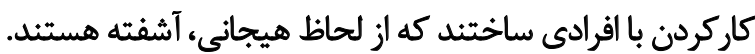

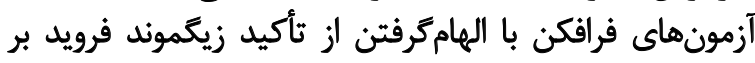




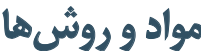

روش يُوهش حاضر از نوع تحليلى و مقطعى است. جامعه ماريه

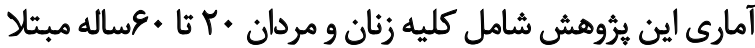

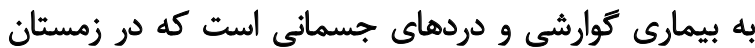

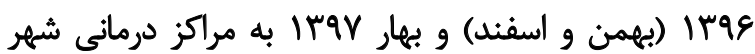

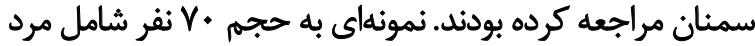

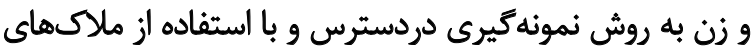

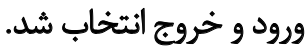

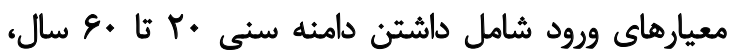

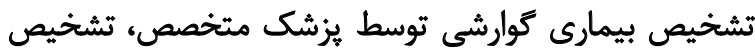

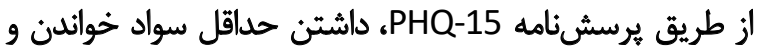

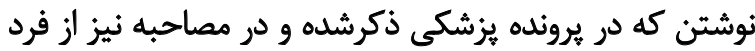

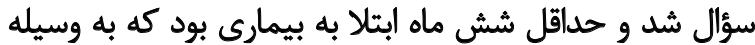

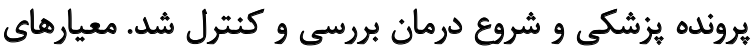

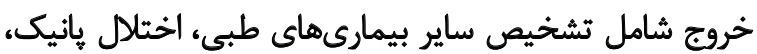

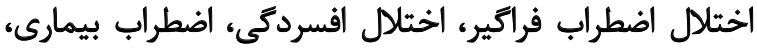

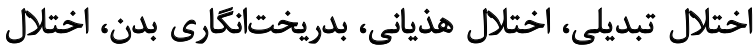

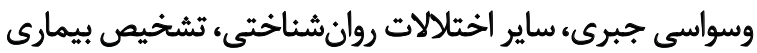

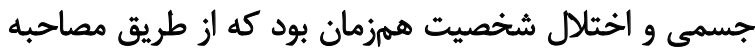

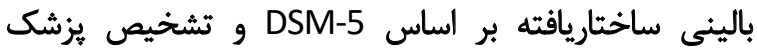
متخصص در يرونده، مورد سنجش قرار كرفت

هيروهش در مراكز درمانى شهر سمنان به مدت • •ه روز انجام

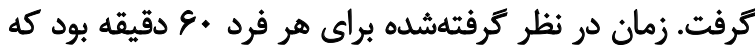

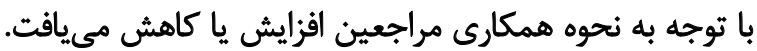

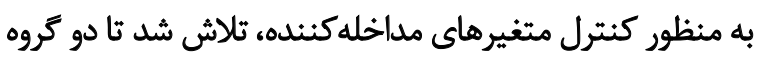

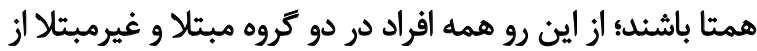

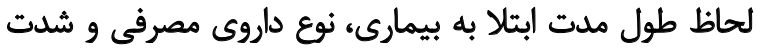

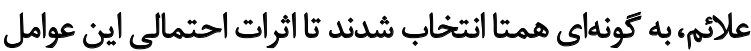

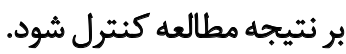

براي جمع آورى دادهها از دو ابزار يثروهشى (يرسشنامه سلامت

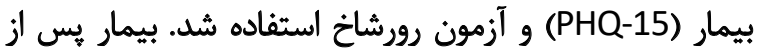

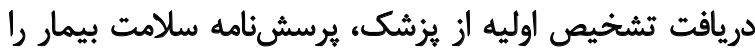

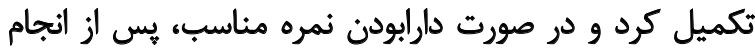

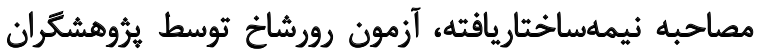

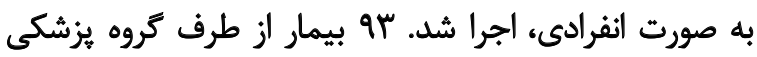

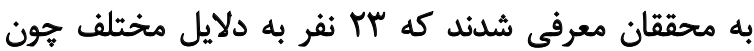

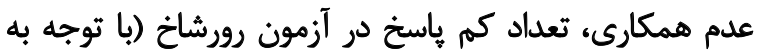

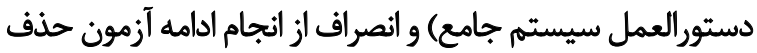

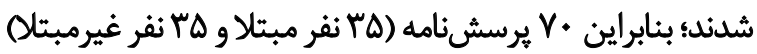
براى تجزيه و تحليل دادهها باقى ماند.
طبق تحقيق بهعملآمده توسط طالعيسند و همكاران كه با

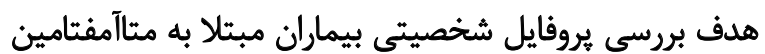

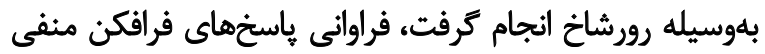

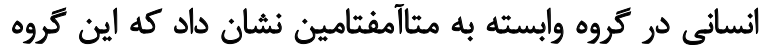

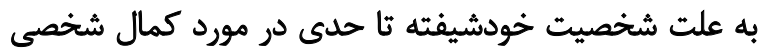

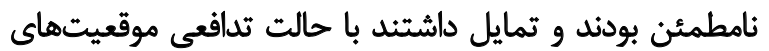

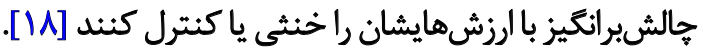

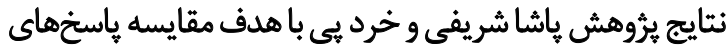

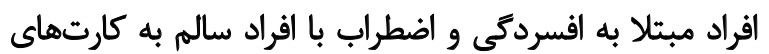

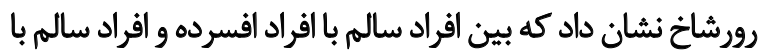

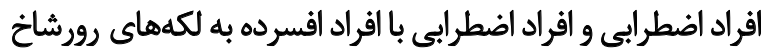

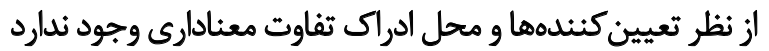

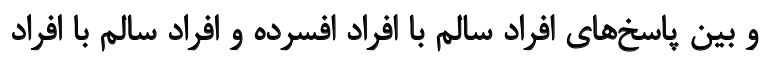

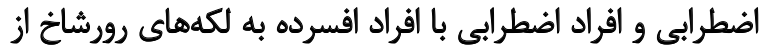
نظر محل ادراك تفاوت معنادار وجود ندارد [ [1 ]ـ.

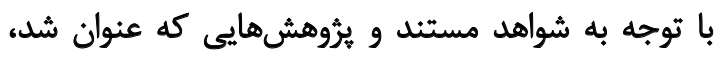

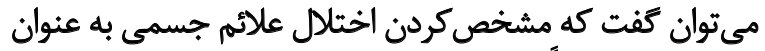

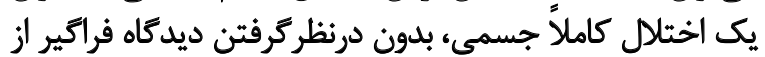

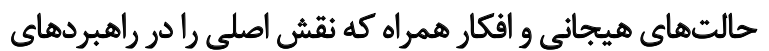

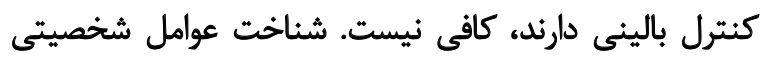

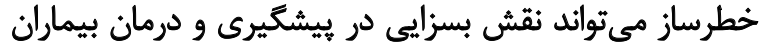

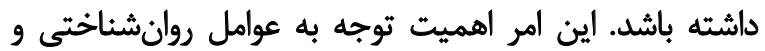

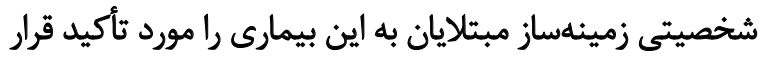

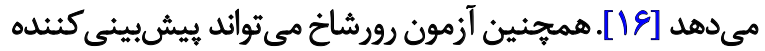

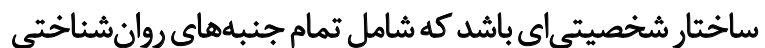

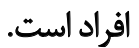

بر اساس اهميت عوامل هيجانى و روانشناختى در اختلال علاثم

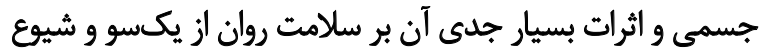

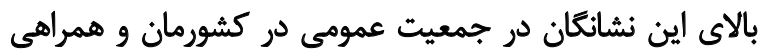

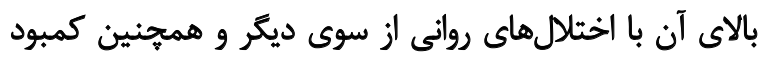

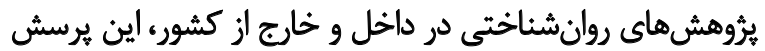

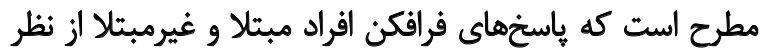

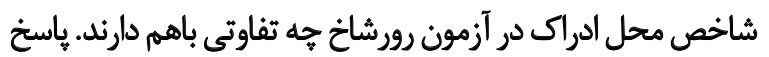

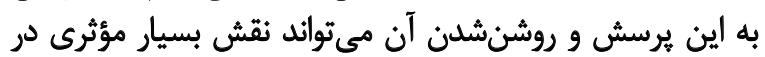
بيشكيرى و فرايند بهبود اين بيماران فراهم كند.

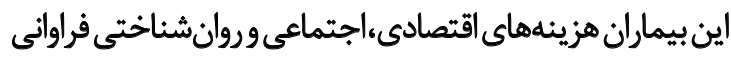

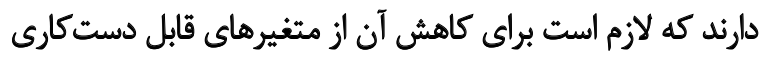

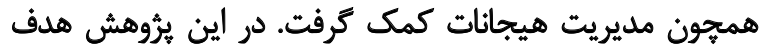

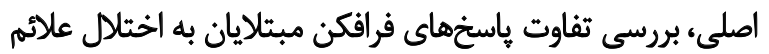

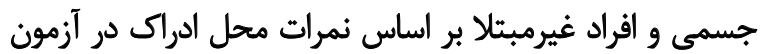

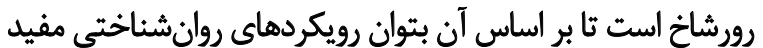

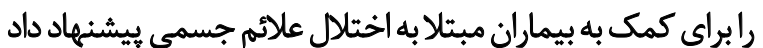

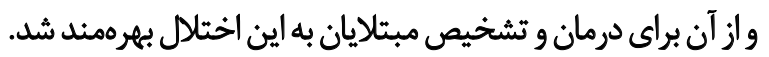




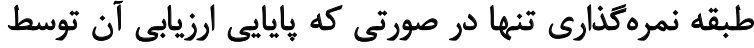

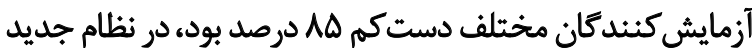

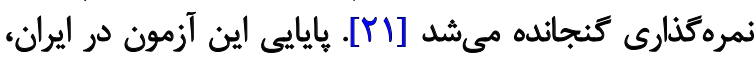

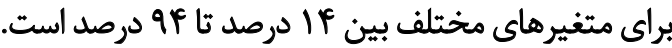

مصاحبه و آزمون با رضايت آكاهانه مراجعين كه هم توسط

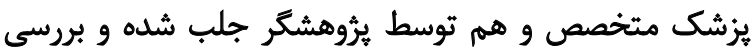

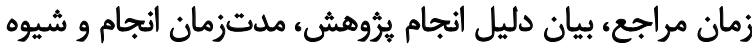

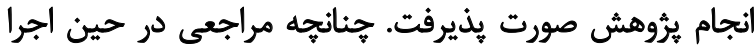

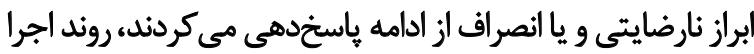

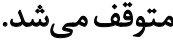

تجزيه و تحليل داده با استفاده از آزمونهاى آمارى توصيفى دئي

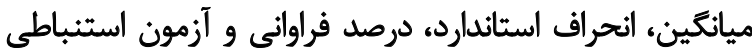

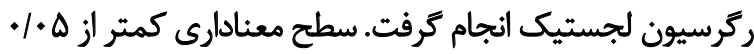

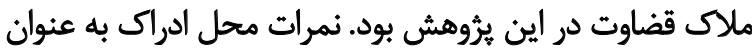

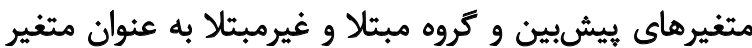

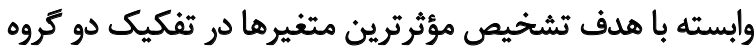

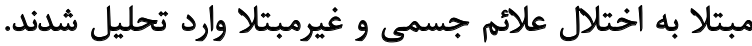

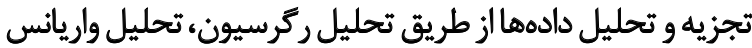

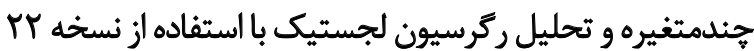
نرمافزار SPSS جهت تحليل دادهها استفاده شد.

يأثتهها

ويرُكى هاى جمعيتشناختى نمونه در جدول شماره إرائهشده

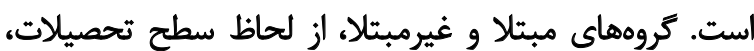

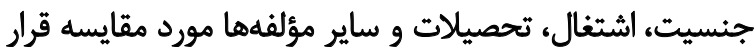

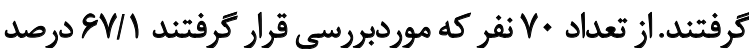

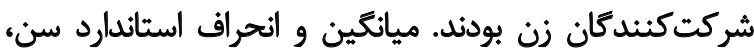

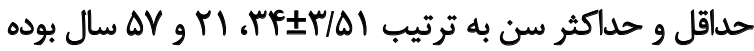

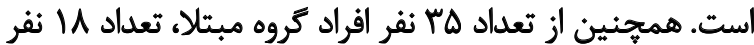

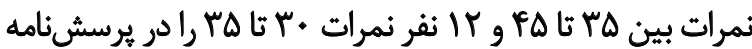

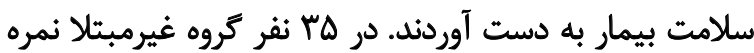

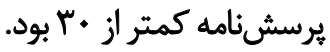

در جدول شماره r، فراوانى شاخص مكان (محل ادراك) آزمون

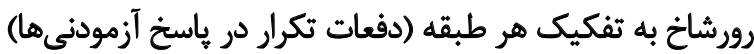

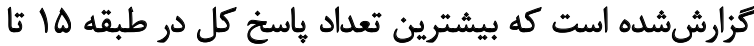

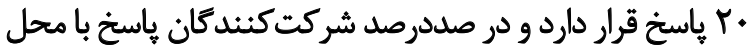

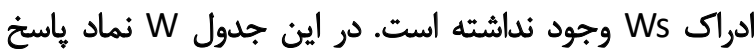

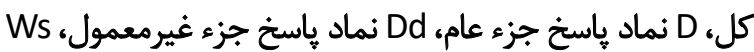

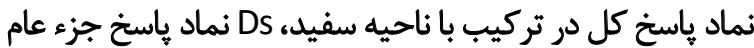

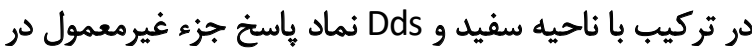
تركيب با ناحيه سفيد است.

به منظور آزمون فرضيه فوق، از تحليل رگرسيون لجستيك

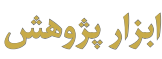

ا. يرسش نامه جسمانى سازي (سلامت بيمار)': نسخه اوليه اين

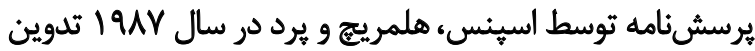

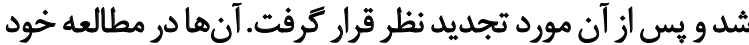

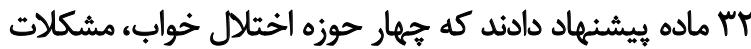

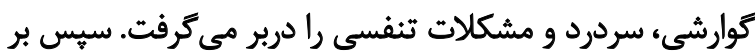

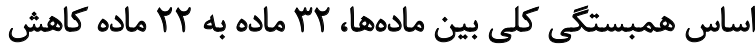

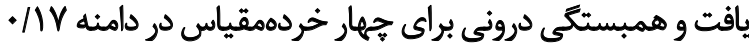

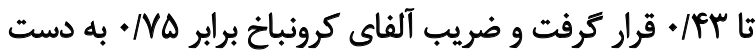

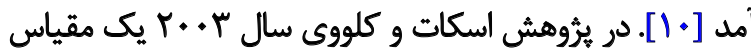

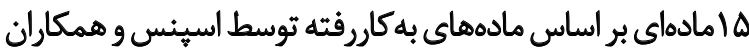
(19/1V)

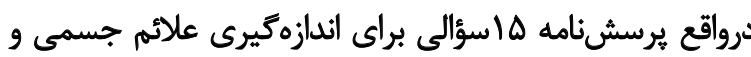
غربالكرى اختلال جسمانى كردن است.

اين مقياس قسمتى از يرسشنامه كامل PHQ بوده و ميزان

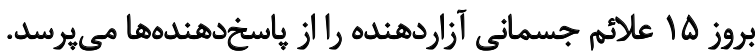

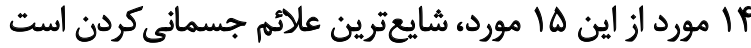

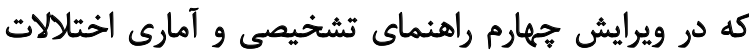

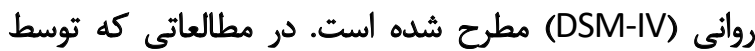

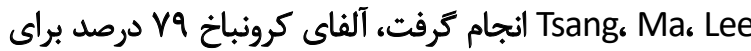

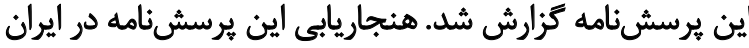

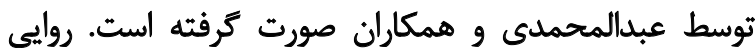

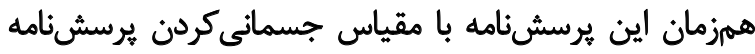

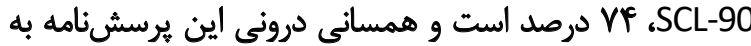

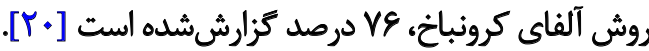

r. آزمون رورشاخ: آزمون رورشاخ از آزمونهاي فرافكن جهان

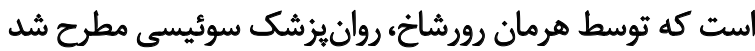

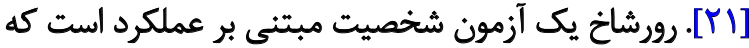

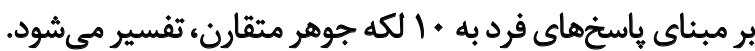

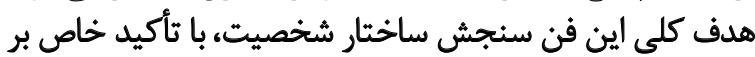

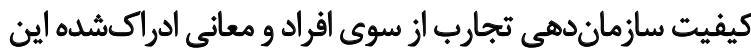
تجارب خاص براى آنهاست [تجارب إنسي

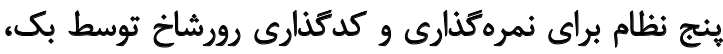

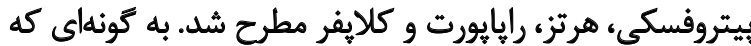

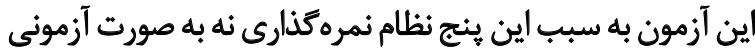

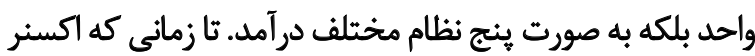

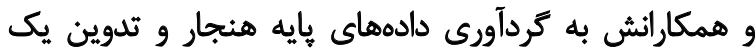

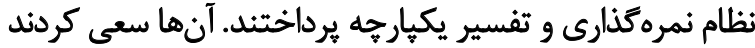

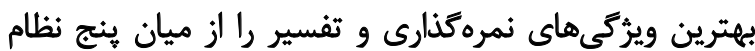

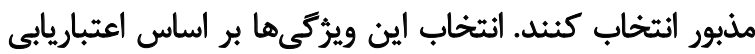

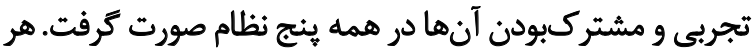

1. Patient Health Questionnaire (PHQ-15) 
جدول ا. ويرُكى هاى جمعيتشناختى نمونه

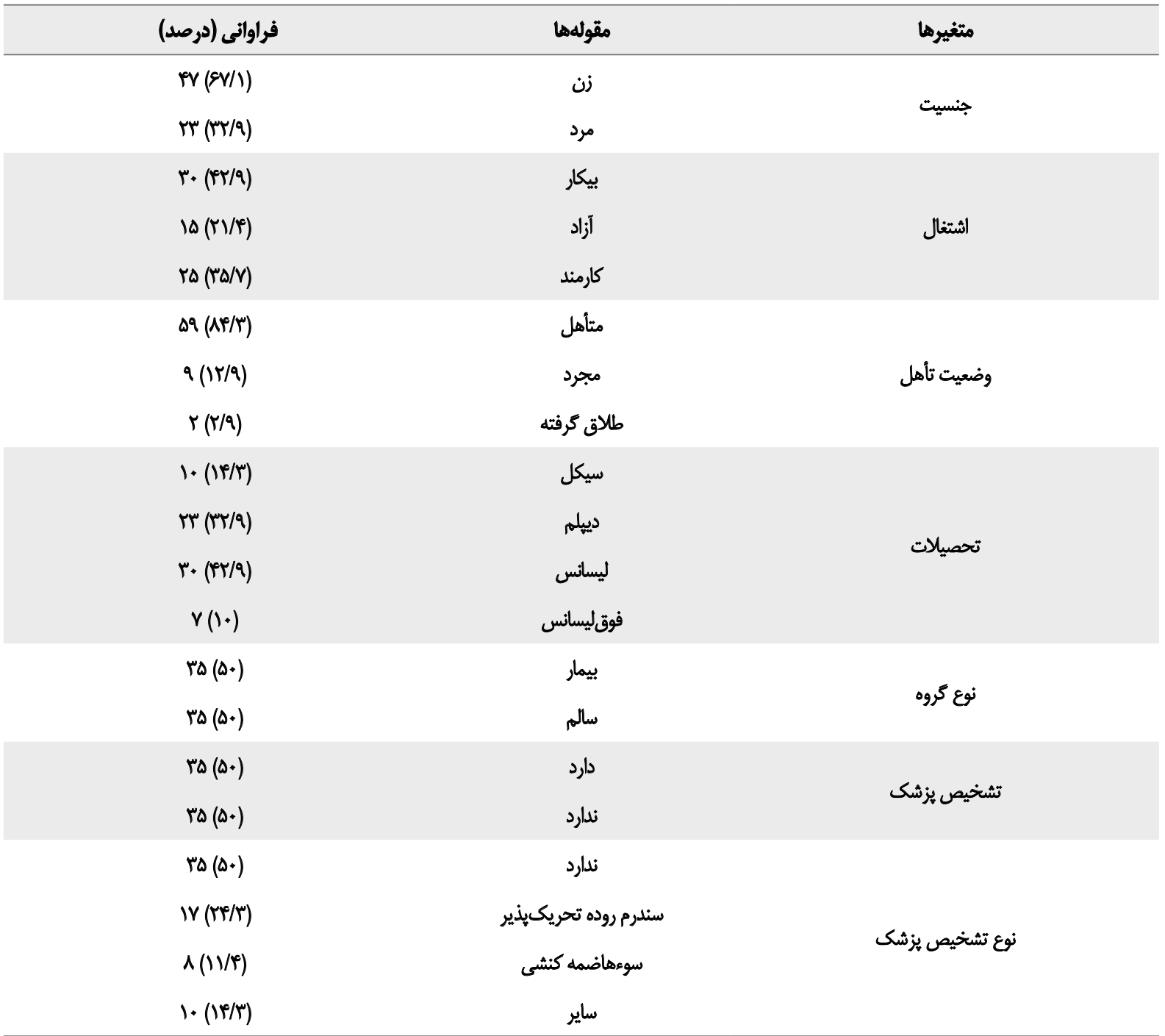

متغيرهاى مستقل راتبيين كرد.

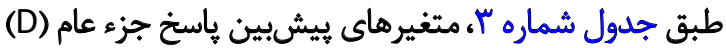

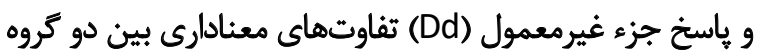

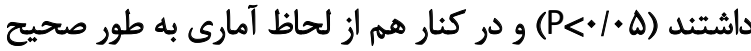

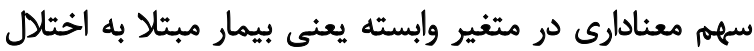

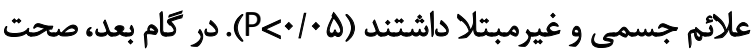

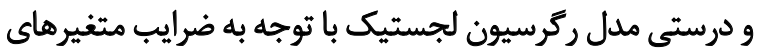

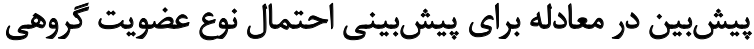

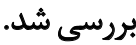

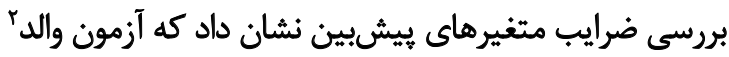

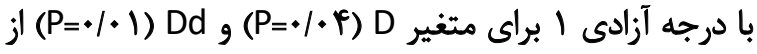

2. Wald test
دومقولهاى با ورود همكام استفاده شد. مدل، شامل ينج متغير

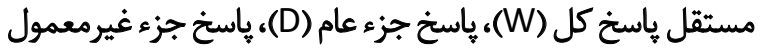

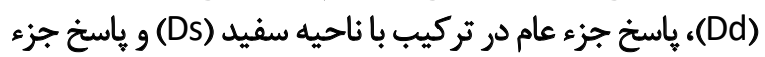

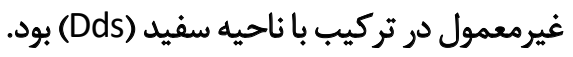
در بررسى نتايج، آزمون نيكويى برازش هوسمر و لمشو به عنوان

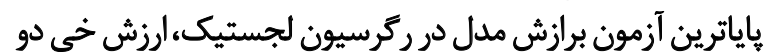

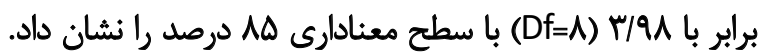

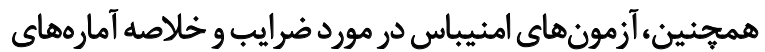

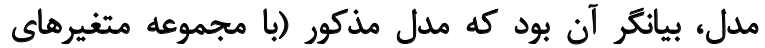

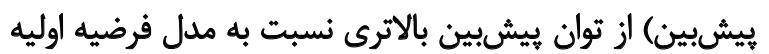

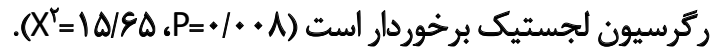
مقادير مجذور كاكس و اسنل (•r/·) و مجذور ناكلكرى

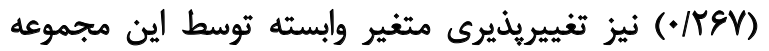


جدول r. توزيع فراوانى (درصد) نمره محل ادراك آزمون رورشاخ

\begin{tabular}{|c|c|c|c|c|c|}
\hline فراوانى (درصد) & & & يراوانى (درصد) & & \multirow{5}{*}{ (R) همجوع ياسخها } \\
\hline$\Delta \cdot(V / / F)$ & - & \multirow{4}{*}{ ياسخ جزء غيرمعمول (Dd) } & $10(M \backslash / F)$ & if & \\
\hline $\operatorname{lr}\left(r_{+}\right)$ & 1 & & $M(\Delta r / q)$ & $r \cdot-10$ & \\
\hline$\Delta(V(N)$ & $r$ & & $\operatorname{IV}(\pi / T)$ & $r \cdot-r$. & \\
\hline $1(1 / 1)$ & $f$ & & $I(1 / f)$ & $r \cdot-r$. & \\
\hline$\lambda\left(11 / R^{*}\right)$ & $\checkmark$ & \multirow{6}{*}{ (W) ياسخ كلى (W) } & $r\left(\mathbb{f} / \mathbb{m}^{m}\right)$ & r & \multirow{6}{*}{ (W) ياسخ كل } \\
\hline$g(N \&)$ & $\wedge$ & & $9(1 \% / 9)$ & $r$ & \\
\hline $1(1 / 4)$ & 9 & & & p & \\
\hline $1(1 / f)$ & 1. & & $\mathbb{H}(\mathbb{N})$ & $\Delta$ & \\
\hline $1(1 / 4)$ & ir & & $10(M / P)$ & 8 & \\
\hline $1(1 / f)$ & 10 & & & & \\
\hline$v(1 \cdot 1 \cdot)$ & ir & \multirow{8}{*}{ ) پاسخ جزء عام (D) } & $1(1 / F)$ & r & \multirow{8}{*}{ (D) باسخ جزء عام } \\
\hline$\lambda(M / 4)$ & if & & $\Delta(V /)$ & $q$ & \\
\hline$r(r / q)$ & 10 & & $f(\Delta / N)$ & $\gamma$ & \\
\hline$r(f / H)$ & 18 & & $\Delta(V /)$ & $\wedge$ & \\
\hline $1(1 / 4)$ & IV & & $\wedge(1 / / 4)$ & 9 & \\
\hline$r(T / q)$ & M & & $A\left(11 / T^{e}\right)$ & 1. & \\
\hline$r(r / q)$ & 19 & & II (WP) & 11 & \\
\hline $1(1 / 4)$ & r. & & $V \cdot(1 \cdot)$. & & \\
\hline $\operatorname{ep}(q) / p)$ & - & \multirow{3}{*}{ تركيب با ناحيه سفيد (Dds) } & $\Delta \cdot(M / P)$ & - & 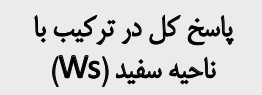 \\
\hline$F(\Delta / V)$ & 1 & & $18(\pi r / q)$ & - & \multirow[t]{2}{*}{ 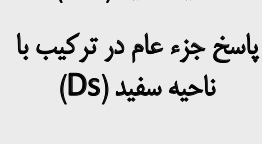 } \\
\hline$r(T / q)$ & $r$ & & $f(\Delta / V)$ & 1 & \\
\hline
\end{tabular}

جدول r. ضرايب متغيرهاى يُيشبين در معادله ركرسيون لجستيك در ييشبينى نوع كروه

\begin{tabular}{|c|c|c|c|c|c|c|}
\hline CL \% 95 & $\operatorname{Exp}(B)$ & $\mathbf{P}$ & Wald & S.E. & B & عامل ييشبين \\
\hline$V+A-+\mid a r$ & - IATY &.$/ 18$ & 1/9ه &.$/ N F$ & -+119 & (W) باسخ كلى (W) \\
\hline$\cdot / 998-\cdot / V+1$ & . /Are & $.1 .+$ & $\varphi / . \varphi$ & .1 .9 &.$-+11 v 9$ & (D) باسخخ جزء عام \\
\hline$\cdot /$ Mre-. $/ \cdot V \Delta$ & . /rua & $.1 \cdot 1$ & $g / I V$ & - IAAP & $-1 / F a$ & ) ياسخ جزء غير معمول (Dd) \\
\hline$r / P Y-\cdot / T q T$ & . $|A A|$ &. Ne & $1 \cdot M$ & - /apf &.$- \mid 181$ & ياسخ جزء عام در تركيب با ناحيه سفيد (Ds) \\
\hline \multirow[t]{2}{*}{$r / T \cdot r-\cdot / N E$} &.$/ N A$ & $.18 \mathrm{~V}$ & .111 &.$/ N Q A$ &.$- / M T H$ & ياسخ جزء غيرمعمول در تركيب با ناحيه سفيد (Dds) \\
\hline & repar & .1 .1 & $\Delta / \& V$ & $1 / \Delta 1$ & r/g.q & علد ثابت \\
\hline
\end{tabular}


در نتيجهاى متفاوت يُؤوهش شريفى وخرد يَى با هدف مقايسه

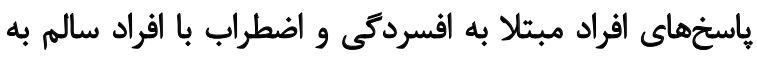

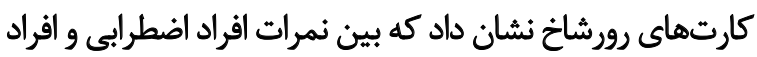

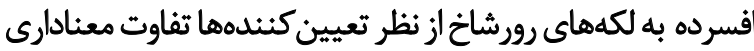

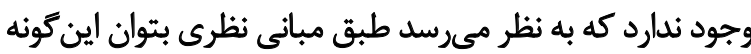

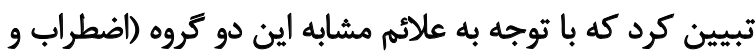

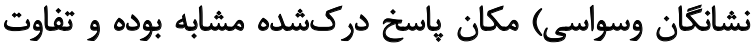
معنادارى نداشته است [19].

\section{نتيجهيكيرى}

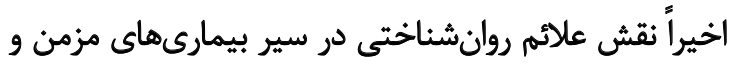

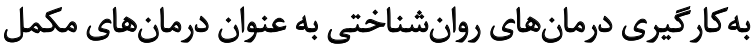

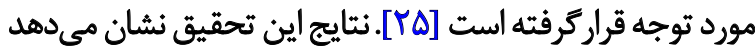

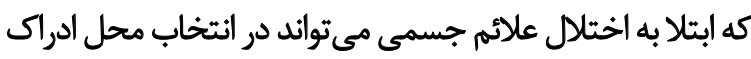

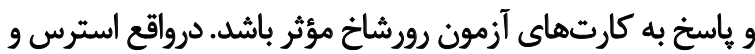

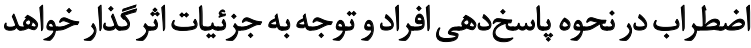

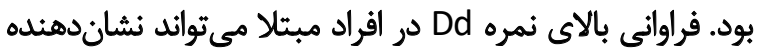

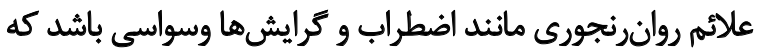

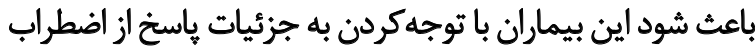

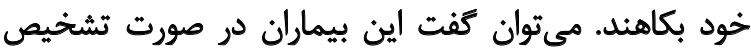

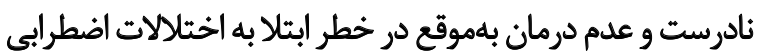

و ورانرنجورى هستند.

نتايج اين يُوهش ضمن تأييد تفاوت باسخها و واهميت آزمون

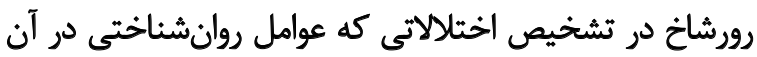

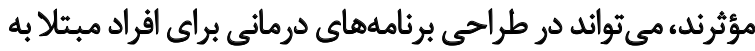

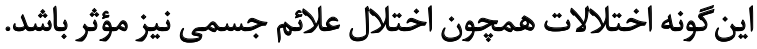

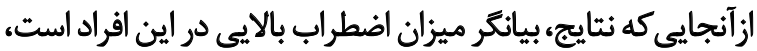

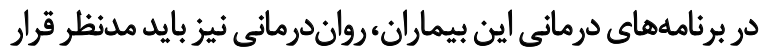

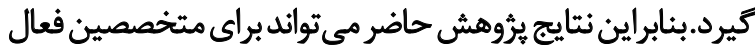

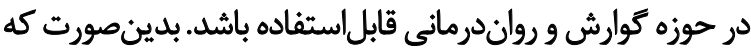

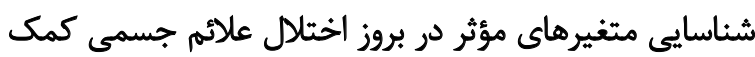

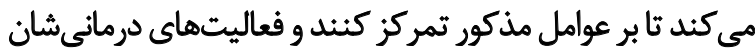

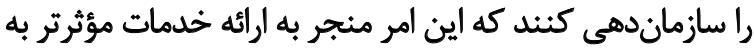

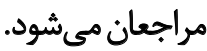

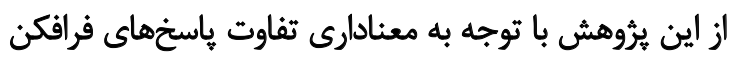

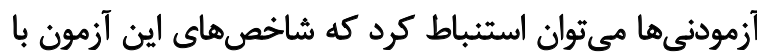

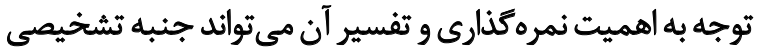

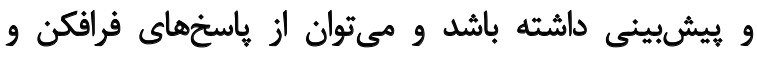

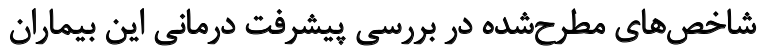

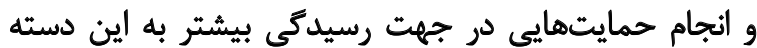

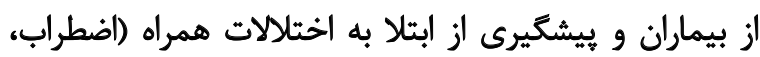
افسردكى، وسواس جبرى) استفاده كرد. از آنجا كه اين يثوهش واجد كاستىهايى است، در
لحاظ آمارى معنادار (داراى مقادير كمتر از ينجصدم) است و اين

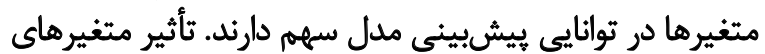

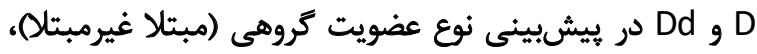

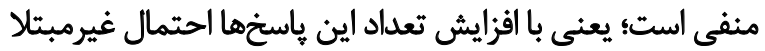

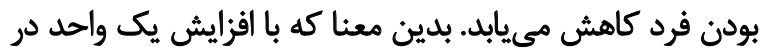

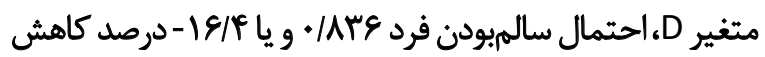

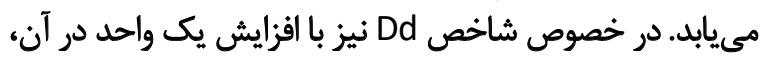

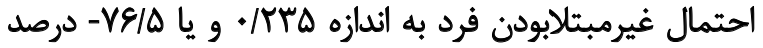

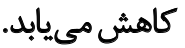
$\stackrel{\leftrightarrow}{\leftrightarrow}$ هدف اين يُؤهش، بررسى ياسخهاى فرافكن افراد مبتلا به

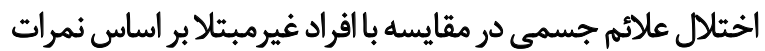

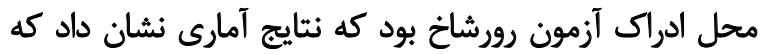

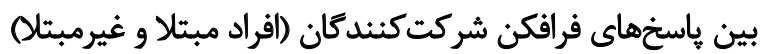

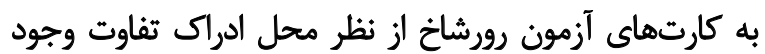

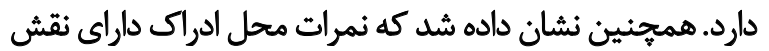
بيشبينى كننده در تفكيك دو كروه مبتلاو غيرمبتئلا هستيند. نتايج تحليل ركرسيون لجستيك نشان داد در ميان نمرات

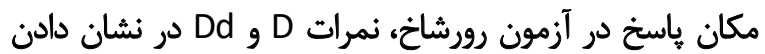

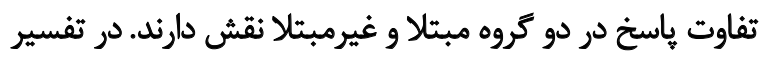

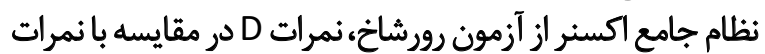

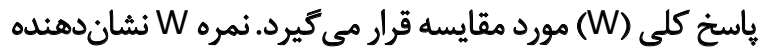

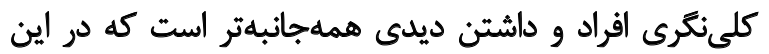

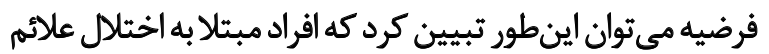

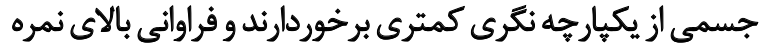

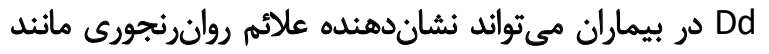

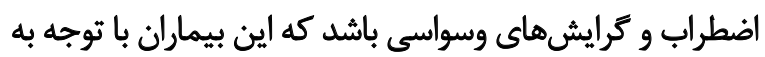
جزئيات ياسخ از اضطراب خود كاستهاند.

در تبيين يافتههاى فوق مىتوان كفت، همانطور كه شعيرى

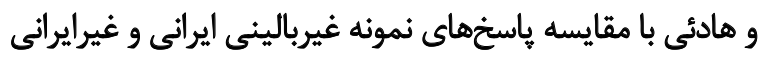

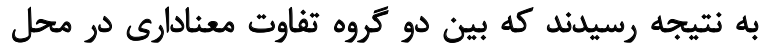

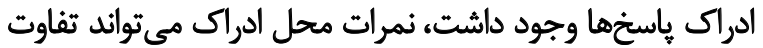

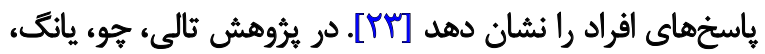

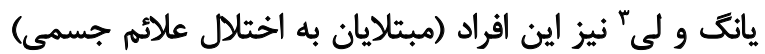

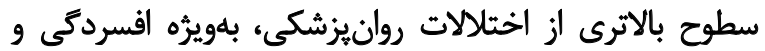

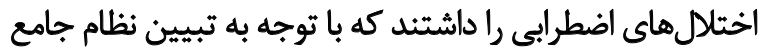

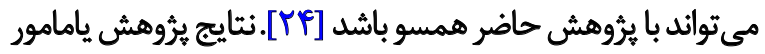

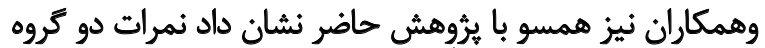

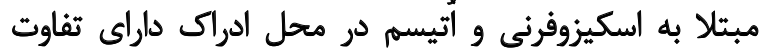

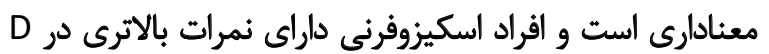

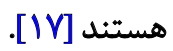


نصيرى (متخصص داخلى) كه با همكارى صميمانه در انجام اين يُروهش، يارىرسان بودند كمال تشكر را دارند.
كعميميافتهها و استنباط نهايى از ثروهش بايد به آنها توجه

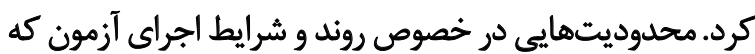

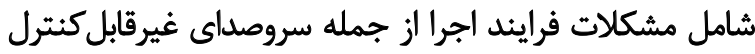

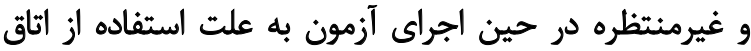

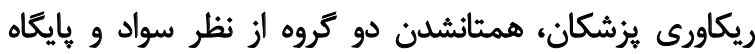

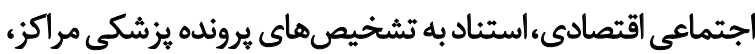

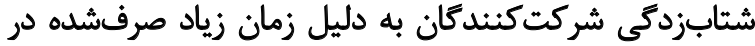

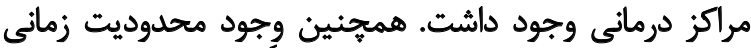

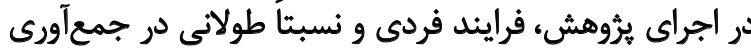

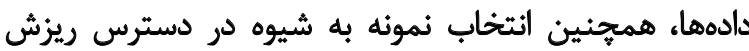

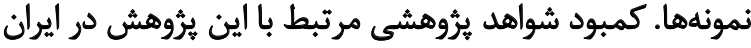

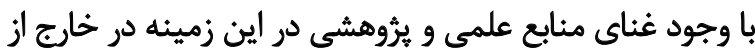
كشور نيز از محدوديتهاى اين يُروهش بودي

بلمنظور كاهش اين كاستى ها بيشنهاد مى شود در يُروهش

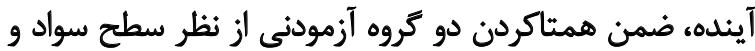

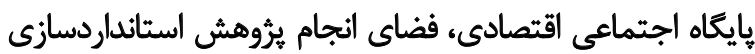

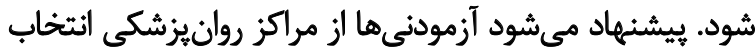

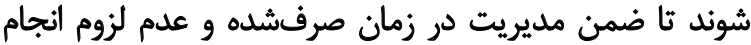

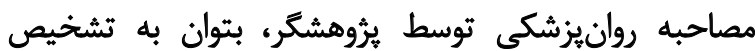
روانيزشك نيز استناد كرد.

مالاحظات اخلاقى

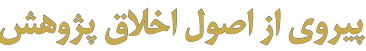

يروهش حاضر توسط كميثه اخلاق دانشكاه علوم يزشكى سمنان تأييد و با شماره توسط كميته

$$
\text { ثُبت رسيد. }
$$

$$
\text { Sto sols }
$$

اين مقاله برترفته از واياننامه كارشناسىارشد نويسنده اول در

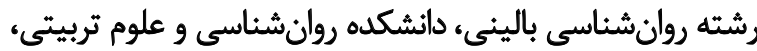
دانشعاه سمنان است.

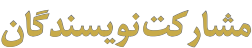

تمام نويسندكان در آمادهسازى اين مقاله مشاركت داشتهاند.

$$
\text { alio vogles }
$$

بنابر اظهار نويسندكان، اين مقاله تعارض منافع ندارد.

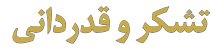

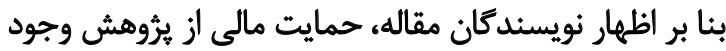

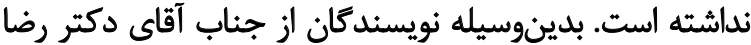
دبيرى (فوقتخصص كوارش و كبد) و جناب آقاى دكتر فرهان آناي دكتر رضان 


\section{References}

[1] Sadrai A, Barati S, Hadadi M,Pormoghadami Z. Compression of psychosomatic disorders among opiate and methamphetamine users. Fourth International Psychosomatic Congress. Islamic Azad University of Khorasgan Branch; 2012.

[2] American Psychiatric Association. Diagnostic and Statistical manuel of mental disorder(DSM) $5^{\text {th }}$ ed. American Psychiatric Association; 2013.

[3] Rief W, Pilger F, Ihle D. Somatization symptoms and hypochondriacal features in the general population. Psychosom Med; 2001: 63:595-602 [DOI:10.1097/00006842-200107000-00012] [PMID]

[4] Barsky AJ, Orav EJ, Bates DW. Somatization increases medical utilization and costs independent of psychiatric and medical comorbidity. Arch Gen Psychiatry. 2005; 62(8):903-10 [DOI:10.1001/archpsyc.62.8.903] [PMID]

[5] Bonvanine IJ, Van Gils A, Janssens KA, Rosmalen JG. Sexual abuse predicts functional somatic symptoms: An adolescent population study. Child Abuse Negl. 2015; 46:1-7. [DOI:10.1016/j.chiabu.2015.06.001] [PMID]

[6] Waller E, Scheidt CE. Somatoform disorders as disorders of affect regulation: A somatoform pain disorder. European Journal of Pain. 2006 7(1):113-9 [DOI:10.1080/09540260500466774] [PMID]

[7] Burton C. Beyond somatisation: A review of the understanding and treatment of medically unexplained physicalsymptom (MUPS). Br J Gen Pract. 2003; 53:231-9.

[8] Rief W, Broadbent E. Explaining medically unexplained symptomsmodels and mechanisms. Clin Psychol Rev. 2007; 27(7):821-41. [DOI:10.1016/j.cpr.2007.07.005] [PMID]

[9] Wollburg E, Voigt K, Braukhaus CH, Herzog A, Lowe B. Construct validity and descriptive validity of somatoform disorders in light of proposed changes for the DSM-5. J Psychosom Res. 2013; 74(1):18-24. [DOI:10.1016/j.jpsychores.2012.09.015] [PMID]

[10] Groben S, Hausteiner C. Somatoform disorders and causal attributions in patients with suspected allergies: Do somatic causal attributions matter? J Psychosom Res. 2011; 70(3):229-38 [DOI:10.1016/j. jpsychores.2010.09.002] [PMID]

[11] Duddu V, Chaturvedi SK, Isaac MK. Amplification and attribution styles in somatoform and depressive disorders-a study from Bangalore, India. Psychopathology. 2003; 36(2):98-103 [DOI:10.1159/000070365] [PMID]

[12] Yamamoto K, Kanbara K, Mutsuura H, Ban I, Mizuno Y, Abe T, et al. Psychological characteristics of Japanese patients with chronic pain assessed by the Rorschach test. Biopsychosoc Med. 2010; 4:20 [DOI:10.1186/1751-0759-4-20] [PMID]

[13] Lwasa K, Ogawa T. Psychological basis of the relationship between the Rorschach texture response and adult attachment: The mediational role of the accessibility of tactile knowledge. J Pers Assass. 2016; 98(3):23846. [DOI:10.1080/00223891.2015.1099540] [PMID]

[14] Exner JE. The rorschach: A comprehensive system Vol. 1. Basic Foundations $4^{\text {th }}$ ed. New York: Wiley; 2003.

[15] Marnat G. Psychological sensory handwriting, Fifth ed. [Hosseini $\mathrm{Na}-$ sab AF, Persian Trans]. Tehran: Arjmand Publishing; 2008.

[16] Eudell-Simmons EM, Hilsenroth MJ. A review of empirical research supporting four conceptual uses of dreams in psychotherapy. Clin Psychol Psychother. 2005; 12(4):255-69.[DOI:10.1002/cpp.445]

[17] Kishimoto N, Yamamuro K, lida J, Ota T, Tanaka SH, Kyo M, et al. Distinctive Rorschach profiles of young adults with schizophrenia and autism spectrum disorder. Neuropsychiatr Dis Treat. 2016; 12:2403-10 [DOI:10.2147/NDT.S116223] [PMID]

[18] Anbari Meibodi N, Talepasand S, Rahimian Boogar I. The projective responses of substance dependants in Rorschach test. Principles of Mental Health. 2014; 16(63):192-9

[19] Khordpey Z. [Comparing the responses of people with depression and anxiety with healthy people to Rorschach cards (Persian)]. [MSc. thesis] Tehran: Islamic Azad University of Tehran; 2014

[20] Kato K, Sullivan PF, Pedersen NL. Latent class analysis of functional somatic symptoms in apopulation-based sample of twins. J Psychosom Res. 2010; 68(5):447-53 [DOI:10.1016/j.jpsychores.2010.01.010] [PMID]

[21] Esposito M, Messina A, Monda V, Bitetti I, Salerno F, Precenzano F, et al. The Rorschach test evaluation in chronic childhood migraine:a preliminary multicenter case- control study. front neurol. 2017; 8:680 [DOI:10.3389/fneur.2017.00680] [PMID]

[22] Weiner IB. Principles of Rorschach interpretation, $2^{\text {nd }}$ ed. Mahwah: ErIbaum; 2003 [DOI:10.4324/9781410607799]

[23] Kashefi N, Shoeiri M, Asghari Moghadam M. Comparison of Rorschach test variables in a non-Iranian non-Iranian sample based on Axner's comprehensive system. J Dev Psychol. 2013; 9(32):410-21.

[24] Ta Li C, Chou Y, Yang K, Yang C, Lee Y, Su T. Medically unexplained symptoms and somatoform disorders: Diagnostic challenges to Psychiatrists. J Chin Med Assoc. 2009; 72(5):251-6 [DOI:10.1016/S1726 4901(09)70065-6]

[25] Sajjadinezhad M, Asgari K, Molavi H, Adibi P. Comparing the effectiveness of cognitive-behavioral stress management,optimism training and medical therapy on somatic symptoms, perceived stress, illness perception and quality of life in patients with ulcerative colitis. Arak Med Univ J. $2016 ; 18(103): 40-54$ 